\title{
IMPORTÂNCIA DE CAPACITAÇÃO SOBRE A PROBLEMÁTICA DO ÁLCOOL: percepção crítica dos profissionais de saúde
}

\author{
TRAINING IMPORTANCE ABOUT ALCOHOL ISSUES: \\ critical perception of health professionals
}

\author{
Sonia Regina Zerbetto', Luciana Dagmar Maciel²
}

\section{RESUMO}

Os programas de capacitação para profissionais de saúde devem ser constantes e enfocar a necessidade de reformulações. O objetivo deste estudo é apreender a percepção destes trabalhadores sobre a importância da capacitação e a implementação do instrumento de rastreamento para álcool e intervenção breve na rede de saúde, após terem realizado tal atividade. Um estudo qualitativo foi realizado no período de setembro a novembro de 2012, com nove trabalhadores de saúde, participantes do processo de treinamento utilizando-se da entrevista semiestruturada e análise de conteúdo Bardin. Os resultados apontaram dificuldades relacionadas às condições objetivas de trabalho, tais como, falta de recursos materiais e humanos, sobrecarga de trabalho e falta de tempo. Além de haver problemas na implementação do instrumento de rastreamento para consumo de álcool e Intervenção Breve no cotidiano laboral, constatou-se, também, a necessidade de investimento dos gestores de saúde na Educação Permanente.

Descritores: Atenção Primária à Saúde; Alcoolismo; Prevenção Primária; Capacitação.

\begin{abstract}
Training programs for health professionals should be constant, focusing on the need to reformulate. The aim of this study is apprehend the perception of health workers about the importance of training and the implementation of the screening tool for alcohol and brief intervention in the health network, after having carried out such activity. A qualitative study was conducted between september to november 2012, with nine health workers, participants in the training process, using the semi-structured interviews and content analysis of Bardin. The results indicated difficulties related work conditions, for example, lack of material and human resources, work overload and lack of time. In addition, there are problems in the implementation of the screening tool for alcohol consumption and Brief Intervention in daily work, it was found, also, the need for investment of health managers in Continuing Education.
\end{abstract}

Descriptors: Primary Health Care; Alcoholism; Primary Prevention; Training. 


\section{Introdução}

A síndrome de dependência alcóolica no Brasil constitui-se em um problema complexo de saúde pública ${ }^{1}$ e requer intervenção em âmbito preventivo, terapêutico e de reabilitação. De acordo com a Classificação Internacional de Doenças (CID-10), este transtorno mental e comportamental devido ao uso de álcool (síndrome de dependência F10.2) caracteriza-se por sintomas comportamentais, cognitivos e fisiológicos, decorrentes do consumo repetitivo do álcool. Exemplifica-se pelo desejo e compulsão ao uso do álcool, falta de controle do consumo, prioridade desta droga em detrimento a outras atividades cotidianas da vida, continuidade do uso apesar de seus danos, além de aumento da tolerância pela droga e/ou abstinência física².

Os dados epidemiológicos do último levantamento nacional apontaram que $50 \%$ de brasileiros maiores de 18 anos consumiram álcool no último ano de 2012; e destes, 17\% foram reconhecidos como dependentes e/ou abusadores ${ }^{3}$.

Para se identificar o padrão de consumo de álcool na dimensão preventiva, utiliza-se o instrumento de rastreamento denominado AUDIT (Alcohol Use Disorders Identification Test), desenvolvido pela Organização Mundial de Saúde (OMS) e validado no Brasil ${ }^{4}$, através de dois estudos em que obtiveram como melhores pontos de corte 7/8, com alta percentagem de especificidade e sensibilidade. Porém, na região sudeste brasileira constatou-se somente a adaptação cultural, definindo-se a pontuação 7 como de corte ${ }^{5}$.

O AUDIT é composto por 10 questões que abordam domínios de quantidade e frequência do beber (as três primeiras perguntas), sintomas de dependência (as três subsequentes) e danos advindos deste uso (as quatro últimas). No referente ao padrão de uso e seu escore, a pessoa pode beber de baixo risco (zero a 7 pontos), beber de risco (8 a 15 pontos), beber abusivo ou nocivo (16 a 19 pontos) e provável dependência (20 a 40 pontos) $)^{2-3,6-7}$.

A Intervenção Breve (IB), aplicada após feedback do AUDIT, tem como objetivo apontar os problemas reais e potenciais, decorrentes do uso do álcool, motivar os indivíduos para mudança de comportamento ${ }^{7}$. Tomando como base a pontuação do AUDIT, o profissional traça a intervenção mais adequada, que pode ser uma orientação, um aconselhamento simples, o estabelecimento de metas (abstemia ou redução de uso) ou o encaminhamento do usuário a um serviço especializado ${ }^{7}$.

Embora estudos nacionais e internacionais apontem a eficácia e eficiência da utilização de instrumentos de rastreamento para 0 consumo de álcool associados à $I^{8-14}$, outros demonstram a existência de dificuldades para implementá-los na atenção primária à saúde, em decorrência da falta de conhecimento e preparo dos profissionais da equipe de saúde ${ }^{8,15}$. Tal fato, demonstra a necessidade de capacitações e treinamentos constantes, com o objetivo de rever conceitos e atitudes dos trabalhadores de saúde frente aos usuários de álcool ${ }^{16}$.

Portanto, este estudo é relevante, por considerar que uma das estratégias da política de atenção à problemática de álcool e outras drogas envolve a capacitação dos profissionais de saúde da rede de atenção básica. Além disso, eles desempenham papel relevante e posição privilegiada para reconhecimento e intervenção junto aos usuários que consomem bebida alcoólica de maneira problemática e danosa ${ }^{16-17}$.

Diante do exposto, pergunta-se: Qual a importância da capacitação para os profissionais de saúde? Quais são as dificuldades elencadas na implementação de tais ações no cotidiano do trabalho, após desenvolvimento desta atividade, sob a óptica dos trabalhadores de saúde?

Este estudo teve como objetivo geral apreender a percepção dos trabalhadores de saúde sobre a importância da capacitação e a implementação do instrumento de rastreamento AUDIT e IB na rede de saúde, após a realização do treinamento para capacitá-los.

\section{Metodologia}

Estudo de caráter descritivo, através de uma abordagem qualitativa, decorrente de um projeto de capacitação de profissionais de saúde da Atenção Básica sobre a aplicação do instrumento de rastreamento AUDIT e IB, em uma cidade do interior paulista.

Uma das capacitações foi efetuada em 2012, em parceria com a Secretaria Municipal de Saúde e Universidade Federal do município em estudo, e programa de treinamento para temática de álcool e outras drogas da Faculdade de Medicina de uma cidade do Estado de São Paulo. O treinamento foi realizado por uma terapeuta ocupacional e dentista da 
Secretaria Municipal de Saúde, uma docente do Curso de Enfermagem de uma Universidade Federal, ambas pertencentes ao município em estudo e teve como proposta preparar tais participantes para serem multiplicadores desta capacitação.

Este grupo, que foram os participantes desse estudo, consistiu de nove trabalhadores de saúde da rede de Atenção Básica: 1 médico, 1 enfermeiro, 1 auxiliar de enfermagem, 2 agentes comunitários de saúde, 1 dentista, 1 psicólogo e 1 assistente social e 1 fonoaudiólogo.

Os critérios de inclusão estabelecidos foram: participar da capacitação no período estabelecido e estar ainda trabalhando em unidades de saúde. Os critérios de exclusão foram: não ter finalizado o curso, estar em férias ou afastamento do trabalho durante o processo de coleta de dados. Para esta coleta, realizou-se uma entrevista semiestruturada, constituída por uma parte de caracterização dos sujeitos, contendo dados pessoais e profissionais, e as referidas questões norteadoras: Como você avalia seu conhecimento para a realização do AUDIT e IB? Como você tem implementado o AUDIT e a IB em seu cotidiano de trabalho (quando, onde, como)? Quais são suas expectativas e crenças em relação à efetividade e eficácia do AUDIT e IB na rotina de trabalho da atenção primária à saúde? Quais dificuldades você percebe na implementação do AUDIT e IB? Utilizou-se do recurso do gravador durante toda a entrevista, a qual foi realizada após assinatura do Termo de Consentimento Livre e Esclarecido pelos participantes. Esta pesquisa respeitou os preceitos éticos, sendo aprovada pelo Comitê de Ética em Pesquisa em Seres Humanos da Universidade Federal de São Carlos, em 14/08/2012, conforme o parecer n 53021/2012.

As entrevistas ocorreram nos meses de setembro a novembro de 2012, com duração média de 30 minutos. Foram transcritas e analisadas pela técnica de análise de conteúdo temática ${ }^{18}$. Procedeu-se a leitura das entrevistas por diversas vezes em profundidade, linha por linha, com seleção e codificação das unidades de análise (sentenças) alfanumericamente, relacionadas com a importância da capacitação e implementação do instrumento AUDIT e IB no cotidiano do trabalho. Sequencialmente, realizaram-se recortes textuais das informações, agregando-os em unidades de registro, de acordo com os temas correlatos, mesmo conteúdo semântico (unidades de sentido) e relevância implícita. Tais unidades foram reagrupadas tematicamente em categorias empíricas e subcategorias, relacionadas às dificuldades de implementação do instrumento e ações da IB, possibilitando as inferências ${ }^{18}$. No tratamento dos resultados, realizaramse inferências e interpretações respaldadas em literatura, captando-se os conteúdos manifestos e latentes contidos nas entrevistas. As falas foram identificadas com as iniciais $E$ (Entrevistados), seguidas de uma numeração, conforme sequência da realização das entrevistas.

\section{Resultados e discussão}

Quanto às características dos participantes, houve predomínio de participantes do sexo feminino (oito), quatro na faixa etária entre 29 a 39 anos, seis católicos, três atuantes na área da saúde em um período de tempo de 1 a 4 anos, e cinco em mais de 15 anos. No referente ao tempo de trabalho na unidade de saúde atual, seis dos entrevistados atuavam entre 1 a 4 anos.

A partir dos dados, surgiram duas categorias temáticas: "Conhecimento adquirido na capacitação" e "Implementação do AUDIT e da IB no equipamento de saúde".

\section{Conhecimento adquirido na capacitação}

Os profissionais de saúde referiram apreensão de novos conhecimentos teóricos e práticos, que envolveram a importância e finalidade do AUDIT e da IB, enquanto ferramenta tecnológica na dimensão preventiva. O AUDIT foi reconhecido pelos participantes como instrumento de rastreamento de usuários por apresentarem um consumo de risco ou não. Eles ressaltaram, ainda, que ambos funcionam como ferramentas de orientação e educação em saúde; porém, para que haja efetividade e eficiência das ações, faz-se necessária a credibilidade do profissional, com possibilidade de acompanhamento e monitoramento do usuário.

"É eficaz, funciona. Mas assim, funciona na questão preventiva. Com essa ferramenta eu também pude orientar o que está acontecendo [...]" (E6) 
"A gente faz a captação desses pacientes, se eles estão em risco ou não estão. Acho que é muito importante, principalmente nessa questão da educação (...) é muito efetivo se a gente acredita mesmo e faz os retornos, a manutenção é muito importante e dá um ótimo resultado." (E8)

Os dados corroboram estudos que apontam a intencionalidade de rastreamento do AUDIT associada à prevenção. Os benefícios desta ferramenta possibilitam educação e diálogo com os usuários sobre o consumo de baixo risco e as consequências do uso problemático ${ }^{19-20}$. Os usuários com pontuação de baixo risco, em abstinência ou abstêmios podem se beneficiar das informações, para evitar que futuramente façam um consumo de modo excessivo ${ }^{2}$. Além disso, podem atuar como multiplicadores destas informações.

Os trabalhadores de saúde citaram a relevância de identificar os estágios motivacionais para a mudança ${ }^{\star 1}$ da pessoa que faz uso problemático de álcool, relacionar a quantidade ingerida de determinada bebida alcoólica com a referência de dose padrão**2 de cada tipo de bebida para orientar o usuário do serviço. Destacaram, também, a importância de auxiliar o usuário na compreensão dos danos do álcool no âmbito dos relacionamentos sociais, principalmente no contexto familiar e laboral.

"[...] Aqueles desenhos da dose, aquilo lá facilita para explicar, porque no meu caso, sem o folhetinho, eu não vou saber aplicar ainda não.[...] ajudando as pessoas, consciência que a bebida realmente, quando a gente extrapola, traz consequências futuras né? Não de imediato, mas futuras, quanto a relacionamento, perda de trabalho, família, acho que é isso." (E2)

"[...] a gente já consegue entender em que estágio [estágios motivacionais para mudança] ele está." (E8)

Os relatos corroboraram resultados de estudo em que aponta que quando o profissional se apropria de tais saberes, pode se tornar mais atento ao padrão de consumo de bebida alcoólica de cada usuário, além de planejar ações preventivas específicas e mais individualizadas ${ }^{20}$. Ao contrário do que alguns profissionais acreditam, é possível atender e cuidar das pessoas que fazem uso abusivo de bebida alcoólica nas USFs, através de aconselhamento simples. Tal conduta possibilita a diminuição do consumo e de danos sociais, econômicos, físicos e psíquicos futuros ${ }^{7}$.

Os depoimentos seguintes exemplificam a importância da IB como estratégia auxiliar de motivação, permitindo ao usuário perceber a sua problemática atual, bem como facilitar a sua adesão ao tratamento.

"Ajuda a pessoa entender o problema e aceitar participar do tratamento, né?"

"[...]Então, por que é interessante a IB? Porque ela é um instrumento que vai trabalhar a motivação, né? Ela ajuda na questão da negação, porque o paciente tem que estar motivado pra se tratar, porque enquanto ele não se vê como um problema, ele não consegue enxergar os prejuízos. Então, a IB ajuda no sentido de estar quebrando essa postura de negação. Quando você faz a intervenção breve também, tem aquele momento da balança [balança decisória], né; que vai fazer o paciente estar enxergando a situação que ele está. Às vezes, a pessoa está vivendo, mas ela não consegue perceber tudo isso, só quando ele para e tá diante de um profissional e para pra pensar" (E7)

No entanto, para ocorrer a motivação do usuário, infere-se que a abordagem do profissional junto a ele requer atitude de respeito e sem confrontos de julgamento moral, seja pela primeira vez ou quando já há um vínculo estabelecido. Salienta-se, ainda, que as ações na fase de aconselhamento breve, dependerão do estágio de prontidão para mudança em que o usuário se encontra.

Os relatos supracitados possibilitaram inferir a relevância do papel dos profissionais de saúde na abordagem do usuário, auxiliando-o a compreender e refletir sobre seu padrão de consumo de álcool e sobre os problemas decorrentes deste.

A IB consiste em uma estratégia motivacional para a mudança de comportamento no consumo de bebidas alcoólicas, que possibilita ao profissional orientar o usuário no processo de aceitação de início do tratamento ${ }^{6-7,22 .}$

\footnotetext{
* 1 O modelo Transteórico de Prochaska e DiClemente aborda o processo de prontidão para mudança das pessoas que fazem uso problemático de álcool ou outras drogas. Esse modelo descreve os estágios (pré-contemplação, contemplação, preparação, ação, manutenção, permeando a recaída), com base em conceitos de motivação e mudança, requerendo para cada etapa uma intervenção diferente ${ }^{21}$.

"2 2 Dose padrão é a unidade de medida que define a quantidade de etanol puro contido nas bebidas alcoólicas. A Organização Mundial da Saúde estabelece que uma dose padrão contém aproximadamente 10 a 12 gramas de álcool puro. Por exemplo, em uma lata de cerveja de 330ml contém 12 gramas de álcool puro (CISA-Centro de Informações sobre Saúde e Álcool, 2016, disponível no site http://www.cisa.org.br/artigo/4503/definicao-dose-padrao.php, acessado em 22 jan 2016.
} 
Entretanto, o modo de abordar o usuário e a forma de intervir, principalmente durante uma primeira abordagem do profissional, poderão facilitar a criação de vínculo entre ambos. Nessa relação, os profissionais são reconhecidos como pessoas que fornecem informações confiáveis e de qualidade, tal como a Educação para o álcool, fator que colabora para o processo de aceitação do problema ${ }^{19} \mathrm{e}$, consequente, engajamento e adesão do usuário ao tratamento. A capacitação realizada possibilitou, ainda, que alguns profissionais se sentissem motivados para a busca de novos conhecimentos sobre a temática em questão, pois perceberam sua relevância no âmbito de seu trabalho diário.

"[...] eu procuro também nas minhas leituras, de uma forma geral, eu sempre estou complementando alguma coisa (...)" (E1).

"Além de ter feito a capacitação, eu também já estava estudando, eu tinha procurado livros, textos, artigos na internet sobre isso". (E6)

Tais relatos corroboraram os dados da literatura, quando esta aponta a valorização da Educação Permanente (EP) baseada na problematização, na reflexão crítica da realidade e na transformação da prática de serviços. A EP não se limita somente a treinamentos formais, mas também na busca pessoal de novos conhecimentos, através da articulação da experiência vivenciada no trabalho, família e sociedade a fim de se educar constantemente ${ }^{16,23}$.

Apesar dos benefícios proporcionados pela capacitação, os relatos apontaram a necessidade de melhorias no processo da realização do curso, relacionadas à supervisão e cobrança em relação à implantação do instrumento AUDIT e a IB na rotina de trabalho das unidades de saúde.

"[...] eu acho que tem que ter uma cobrança [...] te tem que dar resultado. [...] pessoas pensando em como estabelecer ou como fazer essa prática realmente acontecer." (E1)

"Eu acho que praticar mais deveria ser parte dessa capacitação [...] sentir mesmo, como se faz, qual a dificuldade que a gente encontra." (E9)

A implementação de tais capacitações e a expectativa de que o conhecimento adquirido seja inserido no cotidiano de trabalho destes profissionais, requer supervisão e acompanhamento dos gestores de saúde do respectivo município. Estas capacitações devem ser abrangentes e incorporadas a um programa de educação permanente sob a responsabilidade destes gestores, fato corroborado pela literatura ${ }^{5}$. A EP deve ser constante e incentivadora para a mudança de atitudes dos profissionais diante dos usuários. Deve contribuir a fim de que estes se sintam tecnicamente mais confiantes e habilidosos para a utilização de procedimentos de IB, e conscientes da importância de seu papel na orientação e apoio ao usuário ao longo do tratamento ${ }^{24}$. Os treinamentos e capacitações desenvolvidos para os profissionais tornam-se efetivos, quando associados a uma reorganização do sistema de saúde e à consciência dos gestores frente à importância de priorizar tais estratégias ${ }^{5,12,16}$.

\section{Implementação do AUDIT e da IB no equipamento de saúde}

Nesta categoria, os profissionais da rede de saúde relataram as estratégias utilizadas para a aplicação do AUDIT e IB, bem como os diversos problemas enfrentados na aplicação e manutenção destes recursos como atividades no cotidiano do trabalho nas Unidades de Saúde da Família (USFs) e Unidades Básicas de Saúde (UBSs).

Uma profissional de saúde sugeriu, como ideia estratégica a ser implementada no cotidiano das unidades de saúde, a criação de um grupo regular de usuários para aplicação do AUDIT e da IB e seu monitoramento.

"Talvez pensar como a gente vem fazendo, por exemplo, com os outros grupos, de gestante, de hipertenso, diabetes, talvez pensar um grupo do AUDIT . [...] nem que fosse pra fazer uma vez por mês, por alguns dias, aplicar esse teste. Não tem uma equipe na dengue? Então teria que ter uma equipe que vai tratar de fazer a manutenção desses casos; se você pegar 15 dias e todo mundo se envolvesse da unidade, você tiraria muita coisa boa [...] você implementaria muito mais ações pra esses usuários, que esporadicamente, alguém se lembra de aplicar o teste e olhe lá!" (E1) 
A ideia de criar um grupo específico para aplicar o instrumento de rastreamento e IB não vai ao encontro dos objetivos e intencionalidade destes recursos, que podem ser aplicados em qualquer grupo constituído. 0 enfoque preventivo possibilita realizar um diagnóstico da dimensão da problemática de álcool no referido território, e auxiliar no planejamento de intervenções específicas ou gerais, corroborando a literatura ${ }^{6-7,19}$. Entretanto, realizar grupos de usuários com problemas relacionados ao álcool pode ser uma estratégia relevante, principalmente se baseada nos fundamentos da Redução de Danos ${ }^{* * 3}$.

As atividades que visam às ações preventivas e terapêuticas realizadas em locais públicos, visitas domiciliares e em grupos de gestantes, hipertensos e diabéticos constituem momentos em que 0 instrumento de rastreamento e IB podem ser usados como complementação do trabalho²2 já desenvolvido ${ }^{14,25}$.

Conforme os relatos, os profissionais utilizaram as situações rotineiras do atendimento para inserir o AUDIT e a IB, tais como, visitas domiciliares, grupos de atendimento e campanhas realizadas na unidade. Eles iniciaram o diálogo com os usuários, abordando temas relacionados à saúde e na sequência introduziram a temática de álcool e o instrumento de rastreamento. Apesar de haver relato de aplicação nos mais diversos cenários, na prática diária do trabalho das unidades, 0 instrumento se limitou ao momento de acolhimento e raramente a visitas domiciliares, predominando a eventualidade. Para alguns profissionais, a aplicação de tal ferramenta deve-se limitar aos ambientes internos e restritos das unidades de saúde.

"[...] quando eu faço a anamnese eu já vou falando, vou perguntando como estão as coisas, aí a pessoa vai falando dos problemas, a pessoa vai me subsidiando e eu pergunto se posso aplicar esse teste.[...] não tenho a intenção de chegar aplicando nas casas ele é mais para aplicar aqui [USF]." (E.1)

"No acolhimento. Na sala de acolhimento que é um lugar mais restrito e às vezes em uma VD [Visita Domiciliar] que a gente vai e consegue fazer a abordagem; a gente aplica 0 questionário, mas é mais difícil. Normalmente a gente aplica aqui na unidade." (E3)

“(...) em uma campanha de vacinação ou em algum evento que tenha, a gente pode estar aplicando porque é uma ferramenta mais pra esse dia; comemoração da semana do idoso, da gestante e tal" (E5)

Os relatos corroboraram os dados da literatura, ao salientar que o modo e a frequência para a aplicação do AUDIT e IB são ainda incipientes nos equipamentos da atenção primária à saúde, embora a utilização destes recursos seja mais recomendada na deteç̧ão precoce e na realização de alguma intervenção para o uso abusivo de álcool ${ }^{12}$. A inserção destes recursos pode ocorrer em qualquer cenário de saúde, em curto período de tempo, durante consultas médicas e de enfermagem, visitas domiciliares, acolhimento e coordenação de grupos ${ }^{19}$.

Ao contrário do que é preconizado na Intervenção Breve, em que se deve realizar as etapas e monitoramento do usuário em condição de uso de risco abusiviv ${ }^{6-7}$; nas unidades, a conduta terapêutica limitou-se apenas em encaminhamento ao médico para que este assumisse a terapêutica, ou então para as instituições especializadas.

"Então a gente pede para que ele passe em uma consulta médica (...) fazer um encaminhamento pra um psiquiatra, um psicólogo, psicoterapia ou CAPS." (E3)

Os depoimentos denotaram predomínio de um modelo de atendimento centrado no médico, direcionado para as especialidades e focado em encaminhamentos. Dependendo da identificação do padrão de consumo de bebida alcoólica, o usuário pode ser atendido e acompanhado por um dos profissionais da equipe de saúde capacitado e treinado. O profissional médico pode ser acionado, caso o usuário apresente condições físicas, psicológicas ou psiquiátricas debilitantes; como, por exemplo, em situação de intoxicação, dependência ou de abstinência alcoólica. As intervenções realizadas pelos profissionais podem ter como metas a redução do consumo ou abstemia do álcool; porém, tais condutas podem ser compartilhadas entre os membros da equipe, conforme evidências científicas ${ }^{20}$. Assim, a aplicação do AUDIT e IB pode ser realizada a qualquer usuário. Faz-se necessário mencionar que os profissionais da Atenção Básica são os mais adequados para desenvolver este trabalho, desde que estejam preparados e capacitados ${ }^{20}$. No âmbito das dificuldades enfrentadas para a manutenção do AUDIT e da IB na rotina de trabalho, após a capacitação, os relatos

***3 Redução de Danos constitui estratégias para minimizar os danos advindos do consumo de substâncias psicoativas, na dimensão da saúde e de seus aspectos sociais e econômicos, sem necessariamente reduzir o consumo delas. 
mencionaram condições objetivas de trabalho desfavoráveis, identificadas na falta de recursos humanos e materiais, além da sobrecarga de trabalho. Para alguns trabalhadores há ainda descrença na efetividade de tais instrumentos na comunidade, bem como impossibilidade de obter resultados positivos.

"A [nome da pessoa], se ausentou, a médica; a gente ficou com a enfermeira, mas assim, meio sem retaguarda, depois sem agentes comunitários; a gente acaba assim, dividindo muito do nosso tempo com muitos afazeres, né?" (E1)

"[...] deveria ter um espaço na unidade com todos os formatos de doses, os copos, devia ter um cantinho na unidade, sabe?" (E2)

"Eu acho que a Intervenção Breve tem pouco impacto, viu? Pra haver um impacto nesses pacientes é preciso uma ferramenta mais contundente do que o AUDIT." (E4)

Os relatos corroboraram as evidências científicas ao salientarem que, no interior da Atenção Básica de Saúde, há constantemente grande relutância dos profissionais na introdução do AUDIT e a IB no cuidado prestado ao usuário ${ }^{8,15-16}$. Tais fatos justificam-se pela carga de trabalho excessiva ${ }^{5,8}$, preconceitos dos profissionais de saúde e descrença destes na possibilidade de melhora dos usuários ${ }^{8,15-16}$ falta ${ }^{22}$ ou alta rotatividade de recursos humanos no serviç $0^{5,26}$. Quanto aos recursos físicos e materiais, a falta de um espaço privativo e de materiais específicos disponíveis para implementar tal ferramenta tecnológica corroborou a literatura ${ }^{8,22,27}$. Este fato pode ser compreendido devido à crença equivocada de que a Estratégia de Saúde da Família constitua uma política de saúde simples e, portanto, não demanda alta tecnologia, o que contribui para implantação de estruturas improvisadas e insuficientes, dificultando o trabalho a ser desenvolvido ${ }^{27}$. Porém, tais recursos não demandam altos custos, podem ser implementados em associação às atividades diárias já preconizadas e realizadas pelo profissional de saúde, em ambientes de sala de espera, consultórios, domicílio do usuário, espaço do serviço entre outros cenários.

Outros fatores apontados pelos profissionais de saúde referiram à falta de tempo, justificada pela sobrecarga de trabalho, resistência à mudança em seu processo de trabalho.

\section{"Mas aqui a gente não tem muito tempo mesmo, né?" (E2)}

A maior dificuldade que eu tenho nesse sentido é que apesar da capacitação ter acontecido ela efetivamente não está na prática da rede. [...]isso tem que se implantar nas UBSs, nas USFs, né, que são onde vai pegar esse pessoal. As UBSs e USFs têm certa resistência em aderir, em realizar mudanças, porque elas já têm uma forma de trabalhar, um esquema de funcionamento. Quando você introduz isso [AUDIT/IB], significa que vão ter um trabalho a mais." (E7)

Revisão sistemática das barreiras e facilidades para implementação dos instrumentos de rastreamento e IB aponta como dificuldades a gestão da carga de trabalho pela equipe de saúde no contexto da atenção primária à saúde, fator desencadeador de limites aos profissionais em relação à capacidade e disposição para assumir responsabilidade de suas tarefas ${ }^{8}$.

Apesar das justificativas dos profissionais de saúde, salienta-se que na prática, a aplicação do questionário de rastreio dura, no máximo, dois a quatro minutos. A sua avaliação e interpretação demandam menos de um minuto e as etapas da IB, como por exemplo, a educação para o álcool demanda menos de cinco minutos e, caso haja necessidade do aconselhamento breve, este requer até quinze minutos ${ }^{6-7}$.

O perfil da população também constituiu um tema apontado, quando da abordagem da questão das dificuldades, pois a característica de usuários atendidos em determinadas unidades é constituída por um elevado número de alcoolistas crônicos, fator que, para os profissionais, inviabiliza a aplicação do AUDIT, requerendo intervenções de dimensão terapêutica e não preventiva.

"A população onde a gente trabalha é de alcoolista crônico e pra haver um impacto nesses pacientes é preciso uma ferramenta mais contundente do que o AUDIT." (E4)

Apesar da IB ser recomendada para os casos de uso de risco ou abusivo, em que a pessoa ainda não apresenta sintomatologia da dependência, a etapa de aconselhamento breve da IB possibilita ao trabalhador de saúde apontar 
claramente os danos físicos, psicológicos e sociais do uso contínuo da substância psicoativa ao usuário ${ }^{12}$. Isto pode sensibilizá-lo e motivá-lo a rever o seu padrão de consumo e a aderir a uma proposta de tratamento ${ }^{6-7}$. Convém salientar que através do escore do AUDIT, o profissional ao dar um feedback pode concomitantemente sensibilizar o usuário sobre o seu padrão de consumo excessivo, mesmo aquele identificado como provável dependente de álcool, reconhecendo a sua autonomia em buscar metas que incluem reduzir ou parar o consumo de álcool, bem como reconhecer a necessidade de iniciar um tratamento.

\section{Considerações Finais}

A pesquisa evidenciou que embora o AUDIT e IB sejam considerados ferramentas tecnológicas eficazes, ainda há dificuldades de sua implementação no cotidiano do trabalho. Enfatizou, também, que a problemática do álcool não está sendo reconhecida na dimensão preventiva, indo na contramão das políticas públicas de saúde do álcool e outras drogas. Tal fato demonstra a importância de acompanhamento e avaliação permanentes dos gestores em relação aos serviços prestados pelas unidades de saúde para obtenção de resultados mais efetivos, investindo em Educação Permanente.

Para a superação das diversas dificuldades que influenciam a implantação de ações de rastreamento do padrão de uso de álcool e da Intervenção Breve, faz-se necessário melhor planejamento e (re) organização do sistema de saúde. Requer, ainda, contratação de mais profissionais de saúde, além de revisão do processo de trabalho para minimizar a sobrecarga de trabalho e a reformulação das estratégias de educação em saúde.

Os resultados possibilitaram demonstrar que ainda há muitos desafios referentes à implementação de estratégias de prevenção ao consumo de álcool a serem superados.

Este estudo teve como limite metodológico, o número restrito de sujeitos de pesquisa entrevistados, requerendo, assim, um estudo mais abrangente, utilizando-se da saturação teórica, com os profissionais de saúde capacitados neste município.

\section{Referências}

1. World Health Organization (CHE) Global status report on alcohol and health. WHO Geneva, 2014.

2. World Health Organization (CHE). International Statistical Classification of Diseases and Related Health Problems 10th Revision (ICD-10)-WHO Version:2016 [Internet]. Geneva: World Health Organization; 2016. Disponível em: http://apps. who.int/classifications/icd10/browse/Content/statichtml//CD10Volume2_en_2016.pdf. Acesso em: 16 Jun 2016.

3. Instituto Nacional de Ciência e Tecnologia para Políticas Públicas de Álcool e Outras Drogas. II Lenad - Levantamento Nacional de Álcool e Drogas-Relatório 2012. [Internet]. São Paulo: Universidade Federal de São Paulo. Unidade de Pesquisa em Álcool e Drogas; 2014. Disponível em: http://inpad.org.br/wp-content/uploads/2014/03/Lenad-II-Relat\%C3\%B3rio.pdf. Acesso em: 16 Jun 2016.

4. Mendéz EB. Uma versão brasileira do AUDIT - Alcohol Use Disorders Identification Test [dissertação]. Pelotas: Universidade Federal de Pelotas; 1999.

5. Moretti-Pires RO, Corradi-Webster CM. Adaptação e validação do Alcohol Use Disorder Identification Test (AUDIT) para população ribeirinha do interior da Amazônia, Brasil. Cad. Saúde Pública. 2011; 27(3):497-509.

6. Babor TF, Higgins-Biddle JC, Saunders JB, Monteiro MG. Audit: The alcohol use disorders identification test: guidelines for use in primary care. $2^{\mathrm{a}}$ ed. Geneva: World Health Organization, 2001.Disponível em: http://www.talkingalcohol.com/ files/pdfs/WHO_audit.pdf. Acesso em: 16 Jun 2016.

7. Babor TF,Higgins-Biddle JC. Brief Intervention: for hazardous and harmful drinking-a manual for use in primary care. Geneva: World Health Organization, 2001. Disponível em: http://apps.who.int/iris/bitstream/10665/67210/1/WHO_MSD_ MSB_01.6b.pdf. Acesso em: 16 Jun 2016.

8. Johnson M, Jackson R, Guillaume L, Meier P, Goyder E. Barriers and facilitators to implementing screening and brief intervention for alcohol misuse: a systematic review of qualitative evidence. J Public Health (Oxf). [Internet]. 2011; 33(3):412-421. Disponível em: http://jpubhealth.oxfordjournals.org/content/33/3/412.long.doi:10.1093/pubmed/fdq095. Acesso em: 16 Jun 2016. 
9. Woolard R, Baird J, Longabaugh R, Nirenberg T, Lee CS, Mello MJ, Becker B. Project Reduce: reducing alcohol and marijuana misuse: effects of a brief intervention in the emergency department. Addict Behav. [Internet]. 2013; 38(3):1732-1739. Disponível em: http://www.sciencedirect.com/science/article/pii/S0306460312003255.doi:10.1016/j. addbeh.2012.09.006. Acesso em: 16 Jun 2016.

10. Winters KC, Fahnhorst T, Botzet A, Lee S, Lalone B. Brief intervention for drug-abusing adolescents in a school setting: outcomes and mediating factors. J Subst Abuse Treat. [Internet]. 2012; 42(3):279-88. Disponível em: http://www. sciencedirect.com/science/article/pii/S0740547211001681.doi:10.1016/j.jsat.2011.08.005. Acesso em: 16 Jun 2016.

11. Moyer VA. Screening and behavioral counseling interventions in primary care to reduce alcohol misuse: U.S. preventive services task force recommendation statement. Ann Intern Med. [Internet]. 2013; 159(3):210-8. Disponível em: http:// annals.org/article.aspx?articleid=1722524.doi: 10.7326/0003-4819-159-3-201308060-00652.Acesso em: 16 Jun 2016.

12. Pereira MO, Anginoni BM, Ferreira NC, Oliveira MAF, Vargas D, Colvero LA. Efetividade da intervenção breve para 0 uso abusivo de álcool na atenção primária: revisão sistemática. Rev. bras. enferm. 2013; 66(3):420-428.

13. Angus C, Scafato E, Ghirini S, Torbica A, Ferre F, Struzzo P, et al. Cost-effectiveness of a program of screening and brief interventions in primary care in Italy. BMC Fam Pract. [Internet]. 2014; 15(26):1-12. Disponível em: http://bmcfampract. biomedcentral.com/articles/10.1186/1471-2296-15-26.doi: 10.1186/1471-2296-15-26 Acesso em: 23 Jul 2015.

14. Haller DM, Meynard A, Lefebvre D, Ukoumunne OC, Narring F, Broers B. Effectiveness of training family physicians to deliver a brief intervention to address excessive substance use among young patients: a cluster randomized controlled trial. CMAJ. [Internet]. 2014; 186(8):e263-72. Disponível em: http://www.cmaj.ca/content/186/8/E263.Iong.doi: 10.1503/ cmaj.131301. Acesso em: 01 Ago 2015.

15. Broyles LM, Rodriguez KL, Kraemer KL, Sevick MA, Price PA, Gordon AJ. A qualitative study of anticipated barriers and facilitators to the implementation of nurse-delivered alcohol screening, brief intervention, and referral to treatment for hospitalized patients in a Veterans Affairs medical center. Addict Sci Clin Pract. [Internet]. 2012; 7(7):1-20. Disponível em: http://ascpjournal.biomedcentral.com/articles/10.1186/1940-0640-7-7. doi: 10.1186/1940-0640-7-7. Acesso em: 02 nov 2013. 16. Moretti-Pires RO, Corradi-Webster CM. Implementation of brief intervention for problematic alcohol use in primary health in the amazon context. Rev. Latino-Am. Enfermagem. 2011; 19(spe):813-820.

17. Tay AT, Peh AL, Tan SN, Chan HN, Guo S, Chan YH. Alcohol use disorders amongst inpatients in a General Hospital in Singapore: estimated prevalence, rates of identification and intervention. Ann Acad Med Singapore. [Internet]. 2016;45(4):138-47. Disponível em: http://www.annals.edu.sg/pdf/45VolNo4Apr2016/V45N4p138Abstract.pdf. Acesso em: 16 Jun 2016.

18. Bardin L. Análise de conteúdo. 7th ed. Portugal:Edições 70; 2011.

19. Jomar RT, Paixão LAR, Abreu AMM. Alcohol Use Disorders Identification Test (AUDIT) e sua aplicabilidade na Atenção Primária à Saúde. Rev. APS. [Internet]. 2012; 15(1):113-17. Disponível em: http://aps.ufff.emnuvens.com.br/aps/article/ view/1467/598. Acesso em: 22 Jan 2013.

20. Lima KCB, Mata RN, Santos GRD, Teixeira GM, Amaral AKM, Botti NCL. Prática preventiva do uso de risco de álcool na atenção primária à saúde. Rev APS. [Internet]. 2014;17(2):229-35. Disponível em: http://aps.ufjf.emnuvens.com.br/ aps/article/view/2279/807. Acesso em: 26 Jan 2016.

21. Figlie NB, Bordin S, Laranjeira R. Aconselhamento em dependência química. 3rd ed. São Paulo:Roca; 2015.

22. Costa PHA, Mota DCB, Cruvinel E, Paiva FS, Ronzani TM. Metodologia de implementação de práticas preventivas ao uso de drogas na atenção primária latino-americana. Rev Panam Salud Publica. [Internet]. 2013; 33(5):325-331. Disponível em: http://www.scielosp.org/scielo.php?script=sci_arttext\&pid=S1020-49892013000500003\&lng=en. http:// dx.doi.org/10.1590/S1020-49892013000500003.Acesso em: 16 Jun 2016.

23. Branquinho NCSS, Bezerra ALQ, Paranaguá TTB, Paulino VCP. Ações de educação permanente no contexto da estratégia saúde da família. Rev enferm UERJ. 2012; 20(3): 312-6.

24. Corradi-Webster C, Minto E, Aquino F, Abade F, Yosetake L, Gorayeb R, Laprega M, Furtado E. Capacitação de profissionais do programa de saúde da família em estratégias de diagnóstico e intervenções breves para o uso problemático de álcool. SMAD, Rev. Eletrônica Saúde Mental Álcool Drog. (Ed. port.). [Internet]. 2005;1(1):1-10. Disponível em: http://www.revistas.usp. br/smad/article/view/38615/41462. http://dx.doi.org/10.11606/issn.1806-6976.v1i1p01-10. Acesso em: 01 Fev 2013.

25. Gonçalves MAS, Ferreira OS, Abreu AMM, Pillon SC, Jezus SV. Estratégias de rastreamento e intervenções breves como possibilidades para a prática preventiva do enfermeiro. Rev.Eletr.Enf. [Internet]. 2011; 13(2):355-60. Disponível em: http://dx.doi.org/10.5216/ree.v13i2.10502. Acesso em: 16 Jun 2016. 
26. Medeiros CRG, Wagner JAG, GlademirS, loná C, PereiraJLA, LechmannSOMF. Arotatividade de enfermeiros emédicos: um impasse na implementação da Estratégia de Saúde da Família. Ciênc. saúde coletiva. 2010;15(Suppl.1):1521-31. 27. Pedrosa ICF, Corrêa ACP, Mandú ENT. Influências da infraestrutura de centros de saúde nas práticas profissionais: percepções de enfermeiros. Cienc Cuid Saude. 2011; 10(1):058-065.

\section{Sonia Regina Zerbetto}

Endereço para correspondência - Rua: Rodovia Washington Luís, n Km 235-SP-310, CEP: 13.565-905, São Carlos, SP, Brasil.

E-mail: szerbetto@ufscar.br Lattes: http://lattes.cnpq.br/1282433435871232

Luciana Dagmar Maciel - lucidmaciel@yahoo.com.br

\section{Enviado em 02 de fevereiro de 2016.} Aceito em 21 de novembro de 2016. 